\title{
Influence of the microenvironment dynamics on extracellular matrix evolution under hypoxic ischemic conditions in the myocardium
}

\author{
ZENAIDA CEAUȘU ${ }^{1,2}$, MANUELA POPA ${ }^{1}$, BOGDAN SOCEA ${ }^{3,4}$, \\ GABRIEL PETRE GORECKI ${ }^{5}$, MARIANA COSTACHE ${ }^{1,6}$ and MIHAI CEAUŞU ${ }^{1}$ \\ ${ }^{1}$ Pathology Department, 'Carol Davila' University of Medicine and Pharmacy, \\ 020021 Bucharest; ${ }^{2}$ Pathology Department, 'Sf. Pantelimon’ Emergency Hospital, 021659 Bucharest; \\ ${ }^{3}$ Department of Surgery, 'Carol Davila' University of Medicine and Pharmacy, 020021 Bucharest; \\ Departments of ${ }^{4}$ Surgery and ${ }^{5}$ Anesthesiology, 'Sf. Pantelimon' Emergency Hospital, 021659 Bucharest; \\ ${ }^{6}$ Pathology Department, University Emergency Hospital, Bucharest 050098, Romania
}

Received September 29, 2021; Accepted October 29, 2021

DOI: $10.3892 / \mathrm{etm} .2022 .11122$

\begin{abstract}
The extracellular matrix (ECM) consists of fibrillary and non-fibrillary components in the extracellular zone, and fulfills structural and signaling roles. Cardiac insult can lead to cardiomyocyte death, which subsequently determines dynamic changes of ECM composition and regulates cellular responses, ultimately contributing to cardiac repair. The present retrospective study on a small batch selected from the database of the Pathology Department of 'Sf. Pantelimon' Hospital aimed to determine which molecules may have a role in the dynamics of ECM using histopathology and immunohistochemistry methods. The study batch was composed of cases with cardiac ischemic conditions who died at various ages of myocardial infarcts. Tissue samples were taken from the myocardium of the left ventricle (anterior and lateral walls), and multiple series of histological sections were produced and analyzed using immunohistochemistry for collagen type I (Col-1), tenascin C (Tn-C), matrix metalloproteinase 9, CD34, and CD68. Col-1 and Tn-C showed variable patterns of fibrillar plexiform network, associated with a high micro-vascular density of newly formed capillaries revealed by CD34, and an interstitial infiltrate with histiocytes demonstrated by CD68 presence. The ECM represents therefore a polymorphic microenvironment with its own dynamics that is in continuous change, involving a large spectrum of heterogenous molecules, which play different roles in myocardium remodeling under hypoxic ischemic conditions.
\end{abstract}

Correspondence to: Dr Bogdan Socea, Department of Surgery, 'Carol Davila' University of Medicine and Pharmacy, 37 Dionisie Lupu Street, 020021 Bucharest, Romania

E-mail: bogdan.socea@umfcd.ro

Key words: extracellular matrix, cardiac microenvironment, interstitial remodeling, immunohistochemistry, cardiac ischemia, myocardial ischemia

\section{Introduction}

Researchers consider the heart to be an organ that regenerates among the last in mammalian systems. It was previously considered as a post-mitotic organ $(1,2)$. Recent theories state that the heart is characterized by cardiomyocyte turnover throughout life, although these properties are insufficient for restoration in cases of heart disease or after heart injury (3).

Once the cardiomyocytes complete their differentiation and interrupt their mitotic activity, which occurs in the first days after birth, the regenerative capacity of the heart suddenly decreases (4-7).

The extracellular matrix (ECM) is composed of fibrillary (fibrillar collagen) and non-fibrillary components (basement membrane, proteoglycans and glycoproteins), which all have structural and signaling functions. The ECM provides support and anchorage for the shape of the cells and regulates cell dynamics (8).

From a spatial point of view, ECM contains two main areas, the basement membrane/pericellular matrix and the interstitial matrix. The interstitial matrix is organized into three interconnected levels as follows: the epimysium that wraps the entire organ, the perimysium that forms major bundles of myofibers and the endomysium that surrounds individual cardiomyocytes $(3,9)$.

The collagen matrix is mainly composed of collagen type I (Col-1; $>80 \%)$ and Col-3 $(>10 \%)$, which are fixed to the basement membranes of the cardiomyocytes via Col-4 and fibronectin (FN) (10). The ECM anchors chemokines, cytokines, growth factors, proteases [such as matrix metallopeptidases (MMPs)], proteases inhibitors [such as tissue inhibitors of metalloproteinases (TIMPs)], and noncoding RNAs (such as microRNAs) $(3,11)$.

The cardiac interstitium is mainly composed of rod-like thick fibers, located both in the epimysium and the perimysium. These fibers are composed of Col-1, while a fine network of fibers is formed by Col-3, which are more prominent in the endomysium (9). 
MMPs represent a large group of zinc-dependent endopeptidases, which are initially synthesized as zymogens (12). The gelatinases MMP-2 and MMP-9 can be found in cardiac myocytes, cardiac fibroblasts and endocardial cells. The activity of MMPs is inhibited by TIMPs (9).

Following cardiac injury and cardiomyocyte death, cardiac repair cascade is initiated by cellular responses, secondary to dynamic changes in the composition of the ECM (13). The restoration process consists of the following three steps: A first phase of inflammation, a second proliferative phase and a final maturation phase. Inflammatory mediators, such as cytokines and chemokines, are subsequently released from the serum into the interstitial matrix, which leads to leukocyte recruitment and activation of the neutrophils. Inflammatory mediators increase vascular permeability, which is followed by extravasation of plasma proteins, including fibrin, fibrinogen and FN. Simultaneously, MMPs expression and activity are increased (3). The consequence is the formation of provisional ECM with abundant growth factors, to which fibroblasts adhere, inducing therefore fibroblast proliferation and transdifferentiation.

Removal of dead cells and ECM residues by phagocytic activity induces the spill of anti-inflammatory mediators, which is useful in solving the inflammatory phase, thus delimiting the transition to the proliferative phase (14).

Mononuclear cells and macrophages secrete growth factors during the proliferative phase of infarct healing. Large amounts of structural ECM proteins synthesized by myofibroblasts and activated by growth factors modulate several important cell functions such as cell survival, proliferation, polarity, differentiation, adhesion and migration. These proteins also serve key roles in matrix assembly and myocardial protection from adverse remodeling. The best-known growth factors active during this phase are the following: Tenascin-C (Tn-C), Tn-X, thrombospondin, secreted protein acidic and rich in cysteine, osteopontin, osteoglycin, periostin and cellular communication network $(13,15,16)$.

Three forms of cardiac fibrosis are recognized, reflecting distinct mechanisms of fibrotic remodeling. These are the replacement fibrosis, interstitial fibrosis and perivascular fibrosis (9). The replacement fibrosis describes the formation of a scar in zones with myocardium necrosis, representing the consequence of a replacement process that is secondary to primary cardiomyocyte lesion, such as following myocardial infarction. Interstitial fibrosis occurs in the case of different injurious stimuli, including a pressure load, metabolic dysfunction and aging (9).

\section{Materials and methods}

Patient selection. From a database of 100 patients with ischemic heart diseases, a small study batch of 10 cases consisting of 5 men and 5 women (age range, 59-89 years; mean age, $74.8 \pm 7.62$ years; sex ratio, 1:1) with myocardial infarcts of various ages were selected for microscopic investigation of the ECM between January 2016 and December 2020. The tissues were collected at autopsy in 'Sf. Pantelimon' Hospital. The present study fulfilled the ethical criteria of the World Medical Association Declaration of Helsinki. All tissue specimens were harvested in accordance with the legislation of our country and the study protocol was previously approved by the Bioethics Committee of St. Pantelimon Hospital. All patients provided written informed consent at the admission in the hospital.

Histopathology investigation. Heart tissue samples were subjected to histopathological examination. The tissues were collected at autopsy from different parts of the left ventricle, such as the anterior and the lateral walls. The samples were fixed in $10 \%$ neutral buffered formalin ( $\mathrm{pH} \mathrm{7)} \mathrm{for} \mathrm{24-48} \mathrm{h}$ at room temperature and paraffin embedded. Tissues were cut into $5-\mu \mathrm{m}$ sections that were stained by hematoxylin and eosin and van Gieson. Hematoxylin and eosin staining is the standard staining for tissues and was performed in order to obtain the permanent microscopic slide. The staining consists of 2 dyes, namely Meyer hemalaun (a base dye) and Gelbich eosin (an acidic dye), which are used to stain in blue-violet the cell nucleus and in red-pink the cell cytoplasm, respectively. For standard hemalaun \& eosin, the staining takes place at room temperature and briefly, the main steps are the following: staining with Meyer hemalaun (10 min), washing in tap water, washing in $1-3 \mathrm{~cm}^{3}$ saturated solution of $\mathrm{LiCO}_{3}$ (a few seconds), staining with eosin (2-3 min), washing in distilled water and ethanol (90\%), dehydration in ethanol (95\%), ethanol (100\%), xylene and mounting the slides. Van Gieson stain is a trichrome stain for tissues. It is composed of 3 dyes: Weigert hematoxylin, picric acid and acidic fuxin. The nucleus stains black, cytoplasm stains yellow and collagen stains red. The stain is used in light microscopy to highlight the connective tissue, particularly collagen fibers. For Van Gieson, the staining takes place at room temperature and briefly, the main steps are the following: staining with Weigert hematoxylin (15 min), differentiation in chlorohydric alcohol (a few seconds), washing in $\mathrm{LiCO}_{3}$, staining with pycrofucsin (30 sec-1 min), washing in acidic water (few seconds), washing in distilled water, dehydration in ethanol, mounting the slides. Multiple sections were prepared per sample and histopathologically examined. Additional slices $(3 \mu \mathrm{m})$ of tissue have also been prepared for IHC analysis.

IHC analysis was performed for Col-1 (clone, Col-1; 1:100; Sigma-Aldrich; Merck KGaA), Tn-C (clone, 49; 1:100; Leica Microsystems GmbH), MMP-9 (clone, 15W2; 1:400; Leica Microsystems GmbH), CD34 (clone, Qbend; ready to use; CellMarque $^{\mathrm{TM}}$ ), CD68 (clone, KP-1; RTU; CellMarque ${ }^{\mathrm{TM}}$ ) using sections placed on glass slides that were previously treated with poly-L-lysine. IHC was performed for $3 \mu \mathrm{m}$-thick sections (formalin-fixed paraffin-embedded). The method used was an indirect tristadial Avidin-Biotin-Complex technique, using a NovoLink Polymer detection system, which utilizes a novel control polymerization technology to prepare polymeric HRP-linker antibody conjugates, according to the manufacturer's specifications (Novocastra). Antigen retrieval technique (enzymatic pre-treatment) was performed, according to the technical specifications from the producer. The steps were as follows: deparaffinization in xylene for $15 \mathrm{~min}$, rehydration in ethanol series (100\%-5 min, 96\%-5 min, 70\%-5 min), washing in PBS, incubation with normal serum (200 $\mu$ l, Cell Marque) for $20 \mathrm{~min}$, incubation with primary antibody overnight, standard labeled streptavidin-biotin complex (ready to use, Cell Marque), washing in carbonate buffer and development 


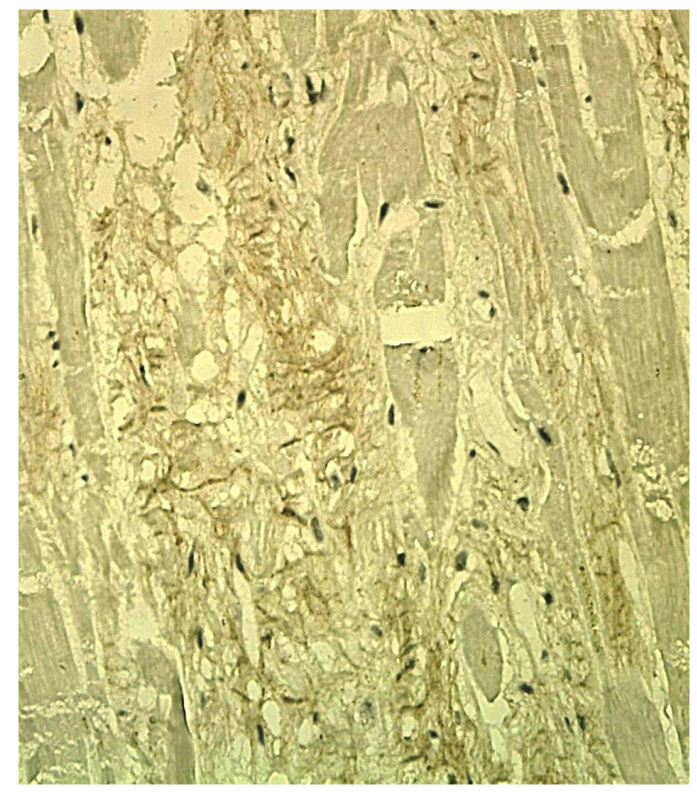

Figure 1. Irregular fragmented fibrilar network of collagen in the extracellular matrix interstitium of hypoxic ischemic myocardium demonstrated by collagen type I staining via immunohistochemistry. Magnification, x200.

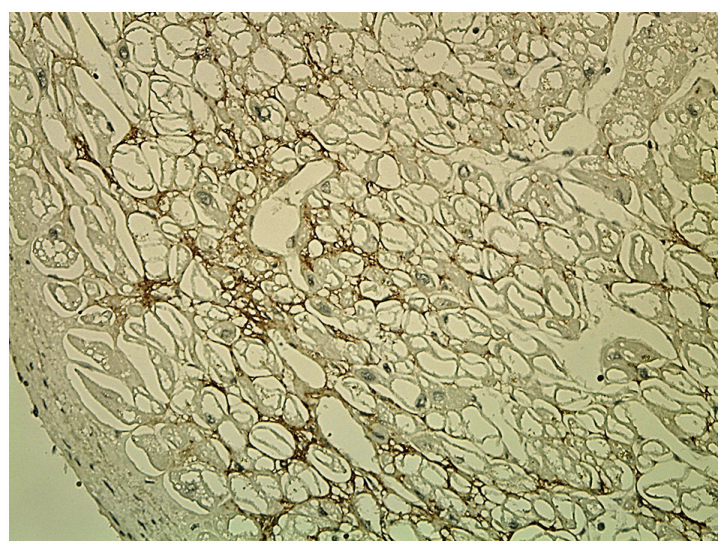

Figure 2. Regular continuous reticular network of tenascin $\mathrm{C}$ in the extracellular matrix of hypoxic ischemic myocardium evaluated by immunohistochemistry. Magnification, x200.

in 3-3'-DAB hydrochloride. All steps were performed at room temperature.

All slides were examined and images were taken using a Leica MC190 HD microscope (Leica Microsystems GmbH; magnification, x100). Images were acquired using an incorporated software program and were further processed and analyzed using Microsoft Office Picture Manager running under Windows 10.

\section{Results}

The histopathological examinations demonstrated various degrees of diffuse or focal interstitial and perivascular fibrosis, due to collagen deposition, along with cardiomyocyte degeneration.

Col-1 staining was focally positive in the interstitium, in the scarring areas and in the residual areas adjacent to the
A

B

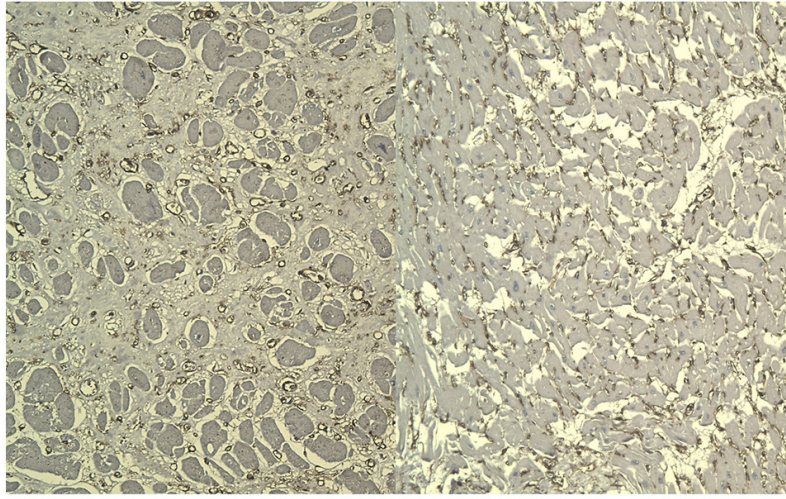

Figure 3. Immunohistochemistry analyses showing (A) a strong diffuse staining for CD34 in newly formed capillary vessels in an old infarcted area and (B) a strong diffuse staining for CD34 in the interstitium of adjacent hypoxic myocardium, revealing a high micro-vascular density. Magnification, $\mathrm{x} 100$

A

B

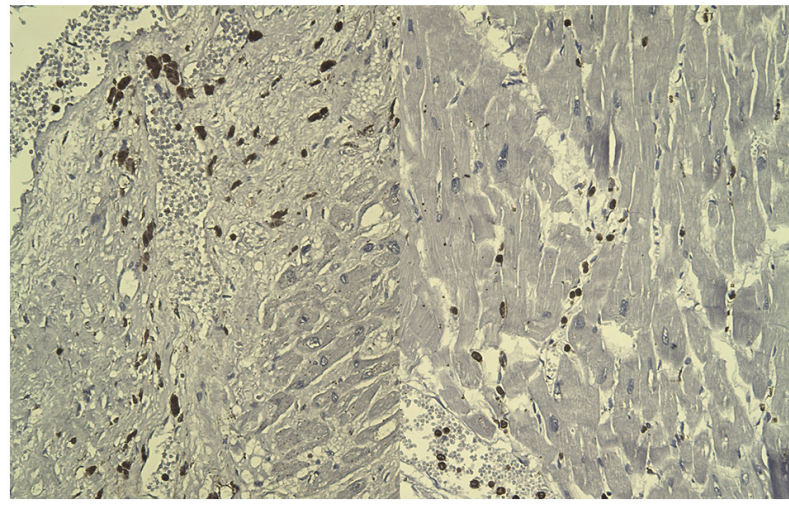

Figure 4. Immunohistochemistry analyses showing (A) a strong staining for CD68 in frequent scattered histiocytes located perivascular in the interstitium of hypoxic ischemic myocardium from an old infarcted area and (B) a strong staining for CD68 in rare interstitial macrophages in a normal adjacent myocardium. Magnification, x200.

myocardial scar. It showed a variable expression in the ECM, with a continuous reticular pattern, in the form of a fibrillar plexiform network (Fig. 1). In recent myocardial infarction, there was no IHC expression of the Col-1 in the ECM.

Tn-C staining was focally positive in subendocardial or subepicardial areas of the ECM. Tn-C was expressed in the ECM in recently infarcted areas and in adjacent residual areas of myocardium. It showed a discontinuous variable network with a reticular pattern (Fig. 2).

MMP9 staining was negative in the ECM, in all layers of the heart, regardless of the age of the infarction.

CD34 staining showed diffuse strong immunoreaction in newly formed capillary vessels, in the adjacent areas of the infarction and in the scarring areas, thus demonstrating a high micro-vascular density (Fig. 3).

CD68 staining was positive in frequent reactive histiocytes, located in interstitial and perivascular areas of the necrotic areas (Fig. 4), but also into the interstitium of the adjacent normal myocardium. 
There was no correlation between the aforementioned markers, which were therefore independent from each other.

\section{Discussion}

Col-1 deposition is difficult to be repaired in conditions of myocardial infarction, as it provides a mechanically strong network for maintaining integrity, minimizing infarct extension and resistance to maladaptive remodeling. The synthesis of Col-1 requires the expression of pro- $\alpha 1$ and pro- $\alpha 2$ collagen chains encoding genes, intracellular assembly of the protein and secretion of procollagen I, which will be cleaved outside the cell and assembled into triple helical fibrils (Col-1). Accumulation of Col-1 can be degraded by degradation of interstitial collagenases and MMP-1, MMP-8, MMP13, MMP2, and membrane type 1 MMP/MMP-14 (17).

The ECM glycoprotein Tn-C is found only in the first stages of embryonic development. Usually, Tn-C is not expressed in the adult heart, but it reappears transiently in conditions of active tissue remodeling in distinct areas of the heart (18). $\mathrm{Tn}-\mathrm{C}$ is considered to be involved in improper left ventricular remodeling, although the exact underlying mechanism of cardiac dysfunction involving Tn-C remains unclear (19).

Cardiac tissue remodeling following myocardial ischemia may be accompanied by an overexpression of $\mathrm{Tn}-\mathrm{C}$ variants. Serum level of Tn-C was reported to be significantly increased in patients with acute myocardial infarction compared with healthy subjects. High serum levels of Tn-C are also correlated with unfavorable prognostic outcome, in the case of left ventricular hypertrophy and major adverse cardiac events (20). A high tissue level of Tn-C was demonstrated in thrombosis, atherosclerotic plaques or stenosis of coronary artery, and bypass-grafts (21-24).

MMP-7 is the metalloproteinase expressed in cardiomyocytes, endothelial cells and macrophages. In animal models of myocardial infarction, the level of MMP-7 increases three-fold at 7 days following infarction, both in ischemic and remote regions $(25,26)$. The high level of MMP-7 activity is associated with an increased risk for major adverse cardiac events, including low survival rate post-myocardial infarction and increased hospitalization period for patients with congestive heart failure (27). Furthermore, high serum MMP-7 level has been demonstrated to be associated with left ventricular structural remodeling in 144 patients with left ventricular hypertrophy (28).

MMP-7 includes a wide variation of target substrates, such as Col-4, FN, Tn-C, connexin-43, peroxiredoxin, laminin and tumor necrosis factor- $\alpha$ (29). MMP-7 can also degrade other MMPs, including MMP-1, MMP-2, and MMP-9, leading to their activation and thus suggesting that MMP-7 might be a direct and indirect regulator for left ventricular remodeling (27). In addition, MMP-7 has major effects on connexin-43 and plays an important role in arrhythmias that appear post infarction (30). Both collagenases (MMP-1) and gelatinases, such as MMP-2 and MMP-9, present high levels in patients with acute ischemic myocardium (31).

Other targets for MMPs also exist that are located intracellularly and are involved in protein degradation, including $\alpha$-actinin, titin and myosin (16). In suffering or remodeled tissues, fragmentation of matrix proteins leads to the release of matrikines. Rapid activation of MMPs in ischemic conditions leads to a rapid matrix fragmentation. However, the functional role of these fragments, which act as bioactive proinflammatory matrikines, remains unclear.

Previous studies demonstrated that in the first $30 \mathrm{~min}$ following coronary ischemia, the serum level of Col-1 fragments increases $(32,33)$. In the infarcted myocardium, fragmentation of constituents of the basement membrane, such as Col-4, and of non-collagenous matrix components also takes place $(16,34)$.

The $\mathrm{CD} 8^{+}$macrophages serve two roles in heart remodeling under ischemic conditions, a fibrogenic role and an angiogenic role. Furthermore, macrophages could also contribute to ECM remodeling by producing MMPs (35).

At an early stage of ischemia, macrophages express certain heterogeneity, gaining regulatory, fibrogenic or angiogenic phenotypes. At a later stage, turnover of macrophages in the ischemic zones depends on proliferation. Subsequent expansion of macrophage population in viable zones is stimulated by chemokines, as a consequence of a high wall stress. Activation of macrophages in the vascularized aria of the myocardium may lead to development and progression of heart failure $(36,37)$.

In summary, the ECM represents a polymorphic microenvironment with its own dynamics that is in a continuous change, involving a large spectrum of heterogeneous molecules, which play different roles in myocardium remodeling under hypoxic ischemic conditions.

\section{Acknowledgements}

Not applicable.

\section{Funding}

No funding was received.

\section{Availability of data and materials}

The data used and/or analyzed in the current study are available from the corresponding author on reasonable request.

\section{Authors' contributions}

$\mathrm{ZC}$ and MiC performed the histological examinations and immunohistochemistry, and provided major contributions in writing the manuscript. $\mathrm{BS}$ and $\mathrm{MaC}$ analyzed and interpreted the data from patient. GPG and MP searched the literature for similar work and articles and contributed to writing the manuscript. ZC and BS confirm the authenticity of all the raw data. All authors read and approved the final manuscript.

\section{Ethics approval and consent to participate}

The present study fulfilled the ethical criteria of the World Medical Association Declaration of Helsinki. This study was approved by the local Bioethics Committee from 'Sf. Pantelimon' Emergency Clinical Hospital (Bucharest, Romania). All patients have previously signed the hospital's standard written informed consent about admission, treatment and a possible future publication of their data. 


\section{Patient consent for publication}

Not applicable.

\section{Competing interests}

The authors declare that they have no competing interests.

\section{References}

1. Bergmann O, Zdunek S, Felker A, Salehpour M, Alkass K, Bernard S, Sjostrom SL, Szewczykowska M, Jackowska T, Dos Remedios C, et al: Dynamics of cell generation and turnover in the human heart. Cell 161: 1566-1575, 2015.

2. Laflamme MA and Murray CE: Heart regeneration. Nature 473: 326-335, 2011

3. Silva AC, Pereira C, Fonseca ACRG, Pinto-do-Ó P and Nascimento DS: Bearing my heart: The role of extracellular matrix on cardiac development, homeostasis, and injury response. Front Cell Dev Biol 8: 621644, 2021

4. Quaini F, Urbanek K, Beltrami AP, Finato N, Beltrami CA, Nadal-Ginard B, Kajstura J, Leri A and Anversa P: Chimerism of the transplanted heart. New Engl J Med 346: 5-15, 2002.

5. Porrello ER, Mahmoud AI, Simpson E, Hill JA, Richardson JA, Olson EN and Sadek HA: Transient regenerative potential of the neonatal mouse heart. Science 331: 1078-1080, 2011.

6. Notari M, Ventura-Rubio A, Bedford-Guaus SJ, Jorba I, Mulero L, Navajas D, Marti M and Raya A: The local microenvironment limits the regenerative potential of the mouse neonatal heart. Sci Adv 4: eaao5553, 2018.

7. Zhu W, Zhang E, Zhao M, Chong Z, Fan C, Tang Y, Hunter JD, Borovjagin AV, Walcott GP, Chen JY, et al: Regenerative potential of neonatal porcine hearts. Circulation 138: 2809-2816, 2018.

8. Chute M, Aujla P, Jana S and Kassiri Z: The non-fibrillar side of fibrosis: Contribution of the basement membrane, proteoglycans, and glycoproteins to myocardial fibrosis. J Cardiovasc Dev Dis 6: 35,2019

9. Frangogiannis $\mathrm{N}$ : The extracellular matrix in ischemic and nonischemicheart failure. Circ Res 125: 117-146, 2019.

10. Bashey RI, Martinez-Hernandez A and Jimenez SA: Isolation, characterization, and localization of cardiac collagen type VI. Associations with other extracellular matrix components. Circ Res 70: 1006-1017, 1992.

11. Fan D, Creemers EE and Kassiri Z: Matrix as an interstitial transport system. Circ Res 114: 889-902, 2014.

12. Visse R and Nagase H: Matrix metalloproteinases and tissue inhibitors of metalloproteinases: Structure, function, and biochemistry. Circ Res 92: 827-839, 2003.

13. Dobaczewski M, Bujak M, Zymek P, Ren G, Entman ML and Frangogiannis NG: Extracellular matrix remodeling in canine and mouse myocardial infarcts. Cell Tissue Res 324: 475-488, 2006.

14. Murphy-Ullrich JE and Sage EH: Revisiting the matricellular concept. Matrix Biol 37: 1-14, 2014.

15. Kong P, Christia P and Frangogiannis NG: The pathogenesis of cardiac fibrosis. Cell Mol Life Sci 71: 549-574, 2014.

16. Frangogiannis NG: The extracellular matrix in myocardial injury, repair, and remodeling. J Clin Invest 127: 1600-1612, 2017.

17. Nong Z, O'Neil C, Le M, Gros R, Watson A, Rizkalla A, Mequanint K, Li S, Frontini MJ, Feng Q and Pickering JG: Type I collagen cleavage is essential for effective fibrotic repair after myocardial infarction. Am J Pathol 179: 2189-2198, 2011.

18. Taki J, Inaki A, Wakabayashi H, Imanaka-Yoshida K, Ogawa K, Hiroe M, Shiba K, Yoshida T and Kinuya S: Dynamic expression of tenascin-C after myocardial ischemia and reperfusion: Assessment by $125 \mathrm{I}$-anti-tenascin- $\mathrm{C}$ antibody imaging. J Nucl Med 51: 1116-1122, 2010
19. Gonçalves I, Acar E, Costantino S, Szabo PL, Hamza O, Tretter EV, Klein KU, Trojanek S, Abraham D, Paneni F, et al: Epigenetic modulation of tenascin $\mathrm{C}$ in the heart: Implications on myocardial ischemia, hypertrophy and metabolism. J Hypertens 37: 1861-1870, 2019.

20. Franz M, Jung C, Lauten A, Figulla HR and Berndt A: Tenascin-C in cardiovascular remodeling: Potential impact for diagnosis, prognosis estimation and targeted therapy. Cell Adh Migr 9: 90-95, 2015

21. Willems IE, Arends JW and Daemen MJ: Tenascin and fibronectin expression in healing human myocardial scars. J Pathol 179: 321-325, 1996.

22. Ballard VL, Sharma A, Duignan I, Holm JM, Chin A, Choi R, Hajjar KA, Wong SC and Edelberg JM: Vascular tenascin-C regulates cardiac endothelial phenotype and neovascularization. FASEB J 20: 717-719, 2006.

23. Kenji K, Hironori U, Hideya Y, Michinori I, Yasuhiko H and Nobuoki K: Tenascin- $\mathrm{C}$ is associated with coronary plaque instability in patients with acute coronary syndromes. Circ J 68: 198-203, 2004

24. Wallner K, Li C, Shah PK, Fishbein MC, Forrester JS, Kaul S and Sharifi BG: Tenascin-C is expressed in macrophage-rich human coronary atherosclerotic plaque. Circulation 99: 1284-1289, 1999.

25. Christia P and Frangogiannis NG: Targeting inflammatory pathways in myocardial infarction. Eur J Clin Invest 43: 986-995, 2013.

26. Cauwe B and Opdenakker G: Intracellular substrate cleavage: A novel dimension in the biochemistry, biology and pathology of matrix metalloproteinases. Crit Rev Biochem Mol Biol 45 351-423, 2010.

27. DeLeon-Pennell K, Meschiari C, Jung M and Lindsey ML: Matrix metalloproteinases in myocardial infarction and heart failure. Prog Mol Biol Transl Sci 147: 75-100, 2017.

28. Zile MR, Desantis SM, Baicu CF, Stroud RE, Thompson SB, McClure CD, Mehurg SM and Spinale FG: Plasma biomarkers that reflect determinants of matrix composition identify the presence of left ventricular hypertrophy and diastolic heart failure. Circ Heart Fail 4: 246-256, 2011.

29. Frangogiannis NG: Matricellular proteins in cardiac adaptation and disease. Physiol Rev 92: 635-688, 2012.

30. Lindsey ML, Escobar GP, Mukherjee R, Goshorn DK, Sheats NJ, Bruce JA, Mains IM, Hendrick JK, Hewett KW, Gourdie RG, et al: Matrix metalloproteinase-7 affects connexin-43 levels, electrical conduction, and survival after myocardial infarction. Circulation 113: 2919-2928, 2006.

31. Danielsen CC, Wiggers H and Andersen HR: Increased amounts of collagenase and gelatinase in porcine myocardium following ischemia and reperfusion. J Mol Cell Cardiol 30: 1431-1442, 1998.

32. Villarreal F, Omens J, Dillmann W, Risteli J, Nguyen J and Covell J: Early degradation and serum appearance of type I collagen fragments after myocardial infarction. J Mol Cell Cardiol 36: 597-601, 2004.

33. Shinde AV and Frangogiannis NG: Fibroblasts in myocardial infarction: A role in inflammation and repair. J Mol Cell Cardiol 10: 74-82, 2014

34. Ceausu Z, Socea B, Dimitriu MC, Predescu D, Constantin VD, Bacalbaşa N, Cîrstoveanu C, Costache M and Ceausu M: Dormant cardiac stem cells: A promising tool in cardiac regeneration. Exp Ther Med 20: 3452-3457, 2020.

35. Chen B and Frangogiannis NG: The role of macrophages in nonischemic heart failure. JACC Basic Transl Sci 3: 245-248, 2018.

36. Ceauşu Z, Socea B, Costache M, Predescu D, Şerban D, Smarandache CG, Pacu I, Alexandru HH, Daviţoiu A, Jacotă-Alexe F, et al: Fibroblast involvement in cardiac remodeling and repair under ischemic conditions. Exp Ther Med 21: 269,2021.

37. Chen B and Frangogiannis NG: Macrophages in the remodeling failing heart. Circ Res 119: 776-778, 2016 